\title{
LACK OF UNIQUENESS FOR WEAK SOLUTIONS OF THE INCOMPRESSIBLE POROUS MEDIA EQUATION
}

\author{
DIEGO CORDOBA, DANIEL FARACO AND FRANCISCO GANCEDO
}

\begin{abstract}
In this work we consider weak solutions of the incompressible 2-D porous media equation. By using the approach of De Lellis-Székelyhidi we prove non-uniqueness for solutions in $L^{\infty}$ in space and time.
\end{abstract}

\section{INTRODUCTION}

The incompressible 2-D porous media equation (IPM) is described by

$$
\rho_{t}+\nabla \cdot(v \rho)=0
$$

where the scalar $\rho(x, t)$ is the density of the fluid. The incompressible velocity field

$$
\nabla \cdot v=0
$$

is related with the density by the well-known Darcy's law [1]

$$
\frac{\mu}{\kappa} v=-\nabla p-(0, g \rho)
$$

where $\mu$ represents the viscosity of the fluid, $\kappa$ is the permeability of the medium, $p$ is the pressure of the fluid and $g$ is acceleration due to gravity. Without lost of generality we will consider $\mu / \kappa=g=1$.

In this paper we study the weak formulation of this system and we construct non trivial solutions with $\rho, v \in L^{\infty}\left(\mathbb{T}^{2} \times[0, T]\right)$ from initial data $\rho(x, 0)=0$. Here $\mathbb{T}^{2}$ is the two dimensional flat torus.

We define a weak solution of $\operatorname{IPM}(\rho, v, p)$ if $\forall \varphi, \chi, \lambda_{1}, \lambda_{2} \in$ $C_{c}^{\infty}\left([0, T) \times \mathbb{T}^{2}\right)$ with $\lambda=\left(\lambda_{1}, \lambda_{2}\right)$ the following identities hold

$$
\begin{array}{r}
\int_{0}^{T} \int_{\mathbb{T}^{2}} \rho(x, t)\left(\partial_{t} \varphi(x, t)+v(x, t) \cdot \nabla \varphi(x, t)\right) d x d t \\
+\int_{\mathbb{T}^{2}} \rho_{0}(x) \varphi(x, 0) d x=0,
\end{array}
$$

$$
\int_{0}^{T} \int_{\mathbb{T}^{2}} v(x, t) \cdot \nabla \chi(x, t) d x d t=0,
$$

Date: December 16, 2009. 


$$
\int_{0}^{T} \int_{\mathbb{T}^{2}}(v(x, t)+\nabla p(x, t)+(0, \rho(x, t))) \cdot \lambda(x, t) d x d t=0 .
$$

For initial data in the Sobolev class $H^{s}\left(\mathbb{T}^{2}\right)(s>2)$ there is localexistence and uniqueness of solutions in a classical sense and global existence is an open problem [4]. It is known the existence of weak solutions, where the motion takes place in the interface between fluids with different constant densities, modeling the contour dynamics Muskat problem [3]. The existence of weak solutions for general initial data is not known. In this context we emphasize that the solutions we construct satisfy

$$
\limsup _{t \rightarrow 0^{+}}\|\rho\|_{H^{s}}(t)=+\infty
$$

for any $s>0$ (see Remark 5.1).

From Darcy's law and the incompressibility of the fluid we can write the velocity as a singular integral operators with respect to the density as follows

$$
v(x, t)=P V \int_{\mathbb{R}^{2}} \Omega(x-y) \rho(y, t) d y-\frac{1}{2}(0, \rho(x)), \quad x \in \mathbb{R}^{2},
$$

where the kernel is a Calderon-Zygmund type

$$
\Omega(x)=\frac{1}{2 \pi}\left(-2 \frac{x_{1} x_{2}}{|x|^{4}}, \frac{x_{1}^{2}-x_{2}^{2}}{|x|^{4}}\right) .
$$

The integral operator is defined in the Fourier side by

$$
\widehat{v}(\xi)=\left(\frac{\xi_{1} \xi_{2}}{|\xi|^{2}},-\frac{\left(\xi_{1}\right)^{2}}{|\xi|^{2}}\right) \widehat{\rho}(\xi) .
$$

This system is analogous to the 2-D surface Quasi-geostrophic equation (SQG) [4], in the sense that is an active scalar that evolves by a nonlocal incompressible velocity given by singular integral operators. It follows that, for Besov spaces, if the weak solution $\rho$ is in $L^{3}\left([0, T] \times B_{3}^{s, \infty}\right)$ with $s>\frac{1}{3}$ then the $L^{2}$ norm of $\rho$ is conserved [27]. This result frames IPM in the theory of Onsager's conjecture for weak solutions of 3-D Euler equations [2],[16]. However there is an extra cancelation, for SQG, due to the symmetry of the velocity given by

$$
\widehat{v}(\xi)=i\left(-\frac{\xi_{2}}{|\xi|}, \frac{\xi_{1}}{|\xi|}\right) \widehat{\rho}(\xi)
$$

that provides global existence for weak solution with initial data in $L^{2}\left(\mathbb{T}^{2}\right)$ [20]. Furthermore, one can find a substantial difference between both systems for weak solutions of constant $\rho$ in complementary domains, denoted in the literature as patches [13]. For IPM the Muskat problem presents instabilities of Kelvin-Helmholtz's type [3] and there is no instabilities for SQG $([21],[8])$. 
The first results of non-uniqueness for the incompressible Euler equations are due to Scheffer [22] and Shnirelman [23] where the velocity field is compacted supported in space and time with infinite energy. The method of the proof we use in this paper is based on understanding the equation as a differential inclusion in the spirit of Tartar [25, 26]. In a ground breaking recent paper De Lellis-Székelyhidi [6] showed that with this point of view the modern methods for solving differential inclusions could be reinterpreted and adapted to construct wild solutions to the Euler equation with finite energy. Our plan was to investigate the scope of this approach in the context of the porous media equation. However it turned out that the situation is different and we have to take different routes at several places which might be of interest for the theory of differential inclusions. We describe them shortly. Firstly, using the terminology of this area that will be recovered in the first section, the special role of the direction of gravity yields certain lack of symmetry in the wave cone $\Lambda$. Moreover the set $K$ describing the non linear constraint belongs to the zero set of a $\Lambda$ convex function. Then it follows that, opposite to Euler, the $\Lambda$ convex hull does not agree with the convex hull and more relevant $K \subset \partial K^{\Lambda}$. This is an obstruction for the available versions of convex integration, the ones based on Baire category [5, 10, 15] and direct constructions [9, 11, 14]. Thus we do not see anyway to use the method to produce solutions to the equation with constant pressure and velocity for some time. Surprisingly, an easy argument shows that the states in $\operatorname{int}\left(\mathrm{K}^{\Lambda}\right)$ can still be used to produce periodic weak solutions starting with $\rho=v=0$. This leaves the difficulty of choosing a proper subset $\tilde{K} \subset K$ with sufficient large $\Lambda$ hull. In general the computation of $\Lambda$ hull might be rather complicated. In this work we argue differently to suggest a more systematic method: Instead of fixing a set and computing the hull, we pick a reasonable matrix $A$ and compute $(A+\Lambda) \cap K$. Then by [10, Corollary 4.19] it is enough to find a set $\tilde{K} \subset(A+\Lambda) \cap K$ such that $A \in \tilde{K}^{c}$ to find what are called degenerate $T 4$ configurations supported in $\tilde{K}^{c}$. To our knowledge this is the first time they are used to produce exact solutions. Then, we are able to choose the set $\tilde{K}$ carefully so that the construction is stable and allow us to solve the inclusion. Our proof of this later fact is related, to the known ways of solving differential inclusions based on T4 configuration [24, 11, 10, 14] but we have made an effort to keep the whole paper self contained. In particular we do not appeal to the general theory of laminates [18, 19, 14] to minimize the amount of new terminology and make the construction more explicit.

The paper is organized as follows. In Section 2 we review the De Lellis-Székelyhidi approach to obtain weak solutions to a nonlinear PDE . Section 3 is devoted to put IPM in this framework, the computation of the wave cone and the corresponding potentials. Section 4 is devoted to the geometry part of the construction and Section 5 to the 
analytic one. In this last section we include some remarks about the nature of our solution as well as why this approach seems to fail for constructing weak solutions compactly supported in space and time.

\section{General strategy and notation}

The strategy to construct wild solutions to a non linear PDE, understanding it as a differential inclusion, builds upon the following main steps. Consider the equation ${ }^{1}$

$$
P(u)=0, \quad u: \mathbb{R}^{n} \rightarrow \mathbb{R}^{m},
$$

where $P$ is a general differential operator.

Tartar framework: Decompose the non linear PDE into a linear equation (Conservation law) $\mathcal{L}(\tilde{u})=0$ and a pointwise constraint $\tilde{u}(x) \in K$. Possibly this requires to introduce new state variables $\tilde{u}$ to linearize the non linearities

$$
P(u)=0 \Longleftrightarrow\left\{\begin{array}{l}
\mathcal{L}(\tilde{u})=0 \\
\tilde{u}(x) \in K
\end{array} .\right.
$$

Specially easy to bring to this framework are equations of the form

$$
\mathcal{L}\left(F_{i}(u)\right)=0
$$

where $F_{i}: \mathbb{R}^{m} \rightarrow \mathbb{R} i=1, \ldots, l$ are some functions possibly non linear. Then one considers new states variables $\tilde{u}_{i}=F_{i}(u)$, which clearly satisfy the conservation law. Then the pointwise constraint amounts to

$$
q_{i}=F_{i}(u) .
$$

Wave Cone: As discovered by Tartar 25] in his development of compensated compactness to such conservation law one can associate a corresponding wave cone $\Lambda_{\mathcal{L}}$, defined as follows:

$$
\Lambda_{\mathcal{L}}=\left\{A \in \mathbb{R}^{m} \times \mathbb{R}^{l}: \exists \xi \text { such that for } z(x)=A h(x \cdot \xi), \mathcal{L}(z)=0\right\},
$$

where $h: \mathbb{R} \rightarrow \mathbb{R}$ is an arbitrary function. That is, the wave cone corresponds to one dimensional solution to the conservation law.

Potential: If $A \in \Lambda_{\mathcal{L}}$ we can build $z: \mathbb{R}^{n} \rightarrow \mathbb{R}^{m} \times \mathbb{R}^{l}$ such that

$$
\mathcal{L}(z)=0 \text { and } z\left(\mathbb{R}^{n}\right) \subset \sigma_{A}
$$

where $\sigma_{A}=\{t A: t \in \mathbb{R}\}$ is the line passing through $A$. The wild solutions are made by adding one dimensional oscillating functions in different directions $A$. For that it is needed to localize the waves. This is done by finding a suitable potential, i.e. a differential operator $D$ such that

$$
\mathcal{L}(D)=0,
$$

\footnotetext{
${ }^{1}$ Here $n$ and $m$ are suitable spaces dimensions that we keep general at this point.
} 
and the plane wave solutions to the conservation law can be obtained from the potential.

Pointwise constraint: In the pointwise constraint one adds the additional features desired for the weak solution (fixed energy, fixed values, etc). This describes a new set $\tilde{K} \subset K$. Then the solution is obtained by two additional steps.

- Find a bigger set $\mathcal{U}$ so that there exists at least a solution to the relaxed system

$$
U_{0} \in \mathcal{U},
$$

with the proper boundary conditions. In practice the set $\mathcal{U} \subset$ $\operatorname{int}\left(K^{\Lambda}\right)$, the so-called $\Lambda$ convex hull of $K$.

- Then one fixes a domain $\Omega$ compact in space and time and in properly chosen subdomains of $\Omega$ one adds infinitely many small perturbations to find a function $U_{\infty} \in K$ a.e $(x, t) \in \Omega$ and $U_{\infty}=U_{0}$ outside of $\Omega$. If $U_{0}$ is a solution to the conservation law, so is $U_{\infty}$. Notice that then $U_{0} \in K$ and to be sure that $U_{\infty} \neq U_{0}$, we need that $U_{0} \notin \tilde{K}$.

There is no standard way to produce the weak solutions from $K^{\Lambda}$ and it is not even always possible. In [6] (for Euler equations) it is enough that $(0,0) \in \mathcal{U}=K^{c}$, which is open in the spaces where the images of the potential lie and that for every state $U \in \mathcal{U}, A_{\tilde{u}} \in \Lambda$ such that the segment which end points $\tilde{u} \pm A \in \mathcal{U}$ and

$$
|A| \geq C \operatorname{dist}(\tilde{u}, K) \text {. }
$$

As discussed in the introduction, this strategy fails in our context and needs to be modified.

\section{Porous media equation}

Here we start by recalling the 2-D IPM system

$$
\left\{\begin{array}{l}
\partial_{t} \rho+\nabla \cdot(v \rho)=0 \\
\nabla \cdot v=0 \\
v=-\nabla p-(0, \rho) .
\end{array}\right.
$$

Our task below is to adapt section 2 to the IPM system.

3.1. Porous media equation in the Tartar framework. Given functions $(\rho, v, q)$ we define the differential operator $\mathcal{L}$ by

$$
\mathcal{L}(\rho, q, v)=\left\{\begin{array}{l}
\partial_{t} \rho+\nabla \cdot q \\
\nabla \cdot v \\
\operatorname{Curl}(v+(0, \rho)) \\
\int_{\mathbb{T}^{2}} v \\
\int_{\mathbb{T}^{2}} \rho
\end{array}\right.
$$


Proposition 3.1. A pair $(\rho, v) \in L^{\infty}\left(\mathbb{T}^{2} \times[0, T], \mathbb{R} \times \mathbb{R}^{2}\right)$ is a weak solution to IPM if and only if we find $u=(\rho, v, q) \in L^{\infty}\left(\mathbb{T}^{2} \times[0, T], \mathbb{R} \times\right.$ $\mathbb{R}^{2} \times \mathbb{R}^{2}$ ) such that,

$$
\left\{\begin{array}{l}
\mathcal{L}(u)=0 \\
q=\rho v
\end{array}\right.
$$

Proof. The two first equations are obvious. The third follows from the Hodge decomposition in $\mathbb{T}^{2}$. Namely consider a vector field $f \in$ $L^{2}\left(\mathbb{T}^{2}\right)$ with $\frac{1}{(2 \pi)^{2}} \int_{\mathbb{T}^{2}} f d x=0$ and curl $f=0$. We claim that the Hodge decomposition implies that $f$ is a gradient field. Namely, every field $f \in L^{2}\left(\mathbb{T}^{2}\right)$ on the torus $\mathbb{T}^{2}$ has a unique orthogonal decomposition

$$
f=\frac{1}{(2 \pi)^{2}} \int_{\mathbb{T}^{2}} f d x+w+\nabla p
$$

such that $\operatorname{div} w=0$. But $\operatorname{curl} f=0=\operatorname{curl} w$. Now we have that $\operatorname{div} w=0$ and $\operatorname{curl} w=0$, then for example by using the formula curl curl $g=-\Delta g+\nabla \operatorname{div} g$ one finds that $\Delta w=0$ and which yields $w=0$. Thus,

$$
f=\frac{1}{(2 \pi)^{2}} \int_{\mathbb{T}^{2}} f d x+\nabla p=\nabla p
$$

It is convenient to write the differential part of $\mathcal{L}$ in a matrix form. For that to each state $u=(\rho, v, q)$ we associate a matrix value function

$$
U(u): \mathbb{R} \times \mathbb{R}^{2} \times \mathbb{R}^{2} \rightarrow \mathbb{M}^{3 \times 3}
$$

by

$$
U(u)=\left(\begin{array}{ccc}
-v_{2}-\rho & v_{1}, & 0 \\
v_{1} & v_{2} & 0 \\
q_{1} & q_{2} & \rho
\end{array}\right)
$$

and the subspace $\mathbb{U} \subset \mathbb{M}^{3 \times 3}$ by $\mathbb{U}=U\left(\mathbb{R}^{2} \times \mathbb{R}^{2} \times \mathbb{R}\right)$.

The following lemma is straightforward.

Lemma 3.1. Let $U=U(\rho, v, q) \in L^{2}\left(\mathbb{T}^{2} \times \mathbb{R}\right)$ with $\frac{1}{(2 \pi)^{2}} \int_{\mathbb{T}^{2}} \rho d x=$ $\frac{1}{(2 \pi)^{2}} \int_{\mathbb{T}^{2}} v d x=0$. Then the following statement holds in the sense of distributions:

$$
\mathcal{L}(u)=0 \Longleftrightarrow \operatorname{Div}(U)=0,
$$

where $\operatorname{Div}\left(a_{i j}\right)=\partial_{j} a_{i j}$ 
3.2. The wave cone. We denote by $\Lambda$ the wave cone related to (IPM).

The reason to write the states in matrix form is that the wave cone is particularly easy to characterize. Namely, we want a plane wave $z(x)=A h(x \cdot \xi)$ to satisfy

$$
\operatorname{Div}(z)=0 .
$$

We obtain that for each $i, h^{\prime}(x \cdot \xi) \xi_{j} a_{i j}=0$, that is

$$
A(\xi)=0 .
$$

In other words $A \in \Lambda$ if and only if $A$ is a singular matrix. Thus $A \in \Lambda \cap \mathbb{U}$ if

$$
-\rho\left(v_{2}^{2}+v_{1}^{2}+\rho v_{2}\right)=0 \Longleftrightarrow \rho=\frac{-|v|^{2}}{v_{2}} \text { or } \rho|v|^{2}=0 .
$$

Observe that fixed $\rho$ this is

$$
v_{2}^{2}+v_{1}^{2}+\rho v_{2}=v_{1}^{2}+\left(v_{2}+\frac{\rho}{2}\right)^{2}-\frac{\rho^{2}}{4}
$$

Thus fixed $\rho$ the corresponding $v \in S\left(\left(0,-\frac{\rho}{2}\right), \frac{|\rho|}{2}\right)$.

3.3. Potentials. The aim of this section is to find a differential operator $D$ such that $\mathcal{L}(D)=0$. We define for $\psi, \varphi: \mathbb{T}^{2} \times \mathbb{R} \rightarrow \mathbb{R}$,

$$
D(\varphi, \psi)=\left(\begin{array}{ccc}
\partial_{x_{1} x_{1}} \psi & \partial_{x_{1} x_{2}} \psi & 0 \\
\partial_{x_{1} x_{2}} \psi & -\partial_{x_{1} x_{1}} \psi & 0 \\
-\partial_{t x_{1}} \psi-\partial_{x_{2}} \varphi & -\partial_{t x_{2}} \psi+\partial_{x_{1}} \varphi & \Delta \psi
\end{array}\right)-\left(\begin{array}{ccc}
\Delta \psi & 0 & 0 \\
0 & 0 & 0 \\
0 & 0 & 0
\end{array}\right)
$$

Or more compactly written

Clearly $D(\varphi, \psi) \in \mathbb{U}$.

$$
\left(\begin{array}{ccc}
-\partial_{x_{2} x_{2}} \psi & \partial_{x_{1} x_{2}} \psi & 0 \\
\partial_{x_{1} x_{2}} \psi & -\partial_{x_{1} x_{1}} \psi & 0 \\
-\partial_{t x_{1}} \psi-\partial_{x_{2}} \varphi & -\partial_{t x_{2}} \psi+\partial_{x_{1}} \varphi & \Delta \psi
\end{array}\right)
$$

Lemma 3.2. Let $\Omega \subset \mathbb{T}^{2} \times \mathbb{R}$ be a bounded open set, $\varphi \in W^{2, \infty}(\Omega)$ and $\psi \in W^{1, \infty}(\Omega)$. Then it holds that

$$
\mathcal{L} D(\varphi, \psi)=0 \text { in } \Omega
$$

in the sense of distributions.

Proof. In our notation we need to obtain that

$$
\operatorname{Div}(D(\varphi, \psi))=0
$$

distributionally. The claim follows since distributional partial derivatives commute. Namely, it holds that

$$
\begin{aligned}
& \text { I: }-\partial_{x_{2} x_{2} x_{1}} \psi+\partial_{x_{1} x_{2} x_{2}} \psi=0, \\
& \text { II: } \partial_{x_{1} x_{2} x_{1}} \psi-\partial_{x_{1} x_{1} x_{2}} \psi=0,
\end{aligned}
$$


III: $\left(-\partial_{t x_{1} x_{1}} \psi-\partial_{t x_{2} x_{2}} \psi+\partial_{t} \Delta \psi\right)+\left(-\partial_{x_{2} x_{1}} \varphi+\partial_{x_{1} x_{2}} \varphi\right)=0$,

as desired. Finally we observe that since the flat torus has no boundary then

$$
\int_{\mathbb{T}^{2}} D(\varphi, \psi)=0
$$

and thus $\mathcal{L} D(\varphi, \psi)=0$.

Remark 3.1. Observe that we are solving $\Delta \psi=\rho$ and then using the PDE to define the rest.

3.4. Plane waves with potentials. In this section we show that for any direction $\Lambda$ with $\rho \neq 0$ we can obtain a suitable potential. It will be more convenient for us to work with saw-tooth functions instead of trigonometric functions. The following proposition is related to [10, Proposition 3.4].

Lemma 3.3. Let $U \in \Lambda$ with $\rho v \neq 0,0<\lambda<1, \epsilon>0, \Omega \subset \mathbb{T}^{2} \times \mathbb{R}$. Then there exists a sequence $u_{N}: \Omega \rightarrow \mathbb{U}$ of piecewise smooth functions such that

- $\mathcal{L}\left(u_{N}\right)=0$

- $\sup \operatorname{dist}\left(u_{N}(x, t),[-(1-\lambda) U, \lambda U]\right) \leq \epsilon$, $(x, t) \in \Omega$

- $\left|(x, t) \in \Omega: u_{N}(x, t)=(-1-\lambda) U\right| \geq \lambda(1-\epsilon)$,

- $\left|(x, t) \in \Omega: u_{N}(x, t)=\lambda U\right| \geq(1-\lambda)(1-\epsilon)$,

- $u_{N} \stackrel{*}{\rightarrow} 0$ in $L^{\infty}\left(\mathbb{T}^{2} \times \mathbb{R}\right)$.

Proof. Let $S \in W^{2, \infty}(\mathbb{R}, \mathbb{R})$ and set

$$
\psi_{N}\left(x_{1}, x_{2}, t\right)=\frac{1}{N^{2}} S\left(N\left(-\frac{v_{1}}{\sqrt{\left|v_{2}\right|}} x_{1}+\sqrt{\left|v_{2}\right|} x_{2}+c t\right)\right)
$$

and

$$
\varphi_{N}\left(x_{1}, x_{2}, t\right)=\frac{1}{N} S^{\prime}\left(N\left(-\frac{v_{1}}{\sqrt{\left|v_{2}\right|}} x_{1}+\sqrt{\left|v_{2}\right|} x_{2}+c t\right)\right) .
$$

Then

$$
D\left(d \varphi_{N}, \psi_{N}\right)=S^{\prime \prime}\left(N\left(-\frac{v_{1}}{\sqrt{\left|v_{2}\right|}} x_{1}+\sqrt{\left|v_{2}\right|} x_{2}+c t\right)\right)\left(\begin{array}{ccc}
-v_{2}-\rho & v_{1} & 0 \\
v_{1} & v_{2} & 0 \\
c \frac{v_{1}}{\sqrt{\left|v_{2}\right|}}-d \sqrt{\left|v_{2}\right|} & -c \sqrt{\left|v_{2}\right|}-d \frac{v_{1}}{\sqrt{\left|v_{2}\right|}} & \rho
\end{array}\right) .
$$

Now the matrix

$$
\left(\begin{array}{cc}
\frac{v_{1}}{\sqrt{\left|v_{2}\right|}} & -\sqrt{\left|v_{2}\right|} \\
-\sqrt{\left|v_{2}\right|} & -\frac{v_{1}}{\sqrt{\left|v_{2}\right|}}
\end{array}\right)
$$


has determinant $-\left(v_{1}^{2}+v_{2}^{2}\right) /\left|v_{2}\right| \neq 0$ and hence defines a bijection of $\mathbb{R}^{2}$. Thus for any $q_{1}, q_{2}$ we can choose $(c, d)$ so that

$$
\left(\frac{v_{1}}{\sqrt{\left|v_{2}\right|}}-d \sqrt{\left|v_{2}\right|},-c \sqrt{\left|v_{2}\right|}-d \frac{v_{1}}{\sqrt{\left|v_{2}\right|}}\right)=\left(q_{1}, q_{2}\right) .
$$

Therefore we have that for any direction in $\Lambda$ with $\rho v \neq 0$ we can obtain a suitable potential.

Next we choose an appropriate function $S$. We consider the Lipschitz 1-periodic functions $S, s: \mathbb{R} \rightarrow \mathbb{R}$ such that $S(0)=0, S^{\prime}=s, s^{\prime}(x)=$ $1-\lambda-\chi_{[\lambda / 2,1-\lambda / 2]}(x)$ for $x \in[0,1]$. We have that $s(1 / 2)=0, s^{\prime}(1 / 2+$ $x)=s^{\prime}(1 / 2-x)$ and $s(1 / 2+x)=s(1 / 2-x)$ if $|x|<1 / 2$.

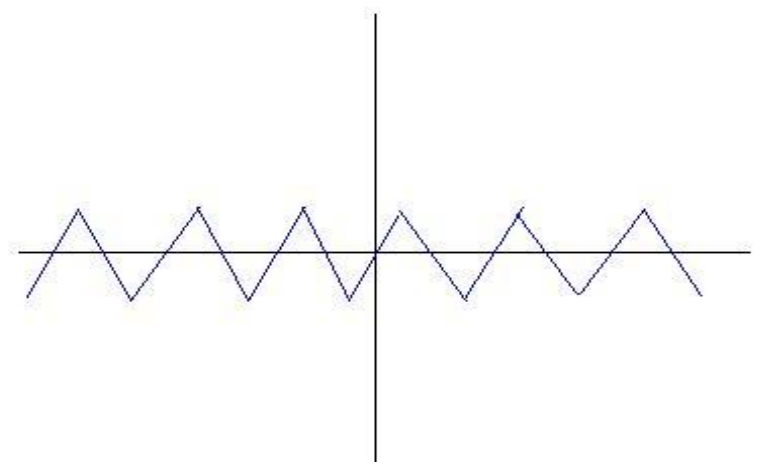

FiguRE 1. The graph of $s$.

We then localize the wave using the potential in the standard way i.e. we define a test functions $\zeta_{\epsilon^{\prime}}$ such that

$$
\left|\zeta_{\epsilon^{\prime}}\right| \leq 1, \quad \zeta_{\epsilon^{\prime}}=1 \text { on } B_{1-\epsilon^{\prime}}(0), \quad \operatorname{supp}\left(\zeta_{\epsilon^{\prime}}\right) \subset B_{1}(0) .
$$

Let $\Omega=B_{1}(0)$ and for a suitable $\epsilon^{\prime}$ we define $u_{N}^{B}=D\left(\zeta_{\epsilon^{\prime}}\left(\varphi_{N}, \psi_{N}\right)\right)$. For a general $\Omega$ consider disjoint balls $B_{r_{k}}\left(x_{k}, t_{k}\right)$ such that

$$
\left|\Omega \backslash \cup_{k} B_{r_{k}}\left(x_{k}, t_{k}\right)\right|>1-\epsilon^{\prime},
$$

and finally we define

$$
u_{N}(x, t)=u_{N}^{B}\left(\frac{x-\bar{x}_{k}}{r_{k}}, \frac{t-t_{k}}{r_{k}}\right) \text { on } x \in B_{r_{k}}\left(x_{k}, t_{k}\right) .
$$




\section{The COnstruCtion}

4.1. Geometric setup. In this section we identify $\mathbb{U}$ with $\mathbb{R} \times \mathbb{R}^{2} \times \mathbb{R}^{2}$. For $A \in \mathbb{U}$ we will use coordinates $(\rho, w, z)$ with $\rho \in \mathbb{R}$ and $w, z \in \mathbb{R}^{2}$. To manipulate the $\Lambda$ cone it will be helpful to introduce the following notation. Along this section we denote by

Similarly,

$$
S_{\rho}=S\left(\left(0, \frac{-\rho}{2}\right), \frac{|\rho|}{2}\right) .
$$

$$
B_{\rho}=B\left(\left(0, \frac{-\rho}{2}\right), \frac{|\rho|}{2}\right) .
$$

The set $K=(\rho, w, \rho w) \subset \mathbb{U}$ defines our pointwise constraint. The strategy in Section 2 requires to find $X_{0} \in(K \backslash \tilde{K}) \cap \operatorname{int}\left(\tilde{K}^{\Lambda}\right)$. However Proposition 5.1 shows that this is not possible. Instead we will find states of the form $(0,0, z) \in \operatorname{int}\left(\tilde{K}^{\Lambda}\right)$. In the next section it will be shown that this is enough to produce a weak solution.

We choose a suitable $\tilde{K} \subset K$. For that we need to introduce the $T 4$ configuration.

Definition 4.1. We say that $C \in \mathbb{U}$ is the center of $T 4$ configuration (degenerate) if there exists $\left\{T_{i}(C)\right\}_{i=1}^{4} \in \mathbb{U}$ such that

a) $C \in\left(\left\{T_{i}(C)\right\}_{i=1}^{4}\right)^{c}$,

b) $C-T_{i}(C) \in \Lambda$.

We say that $A$ belongs to the $T 4$ configuration if $A \in\left[C, T_{i}(C)\right]$ for some $i$. In this case we also set $T_{i}(A)=T_{i}(C)$.

T4 has been the key pieces of subtle versions of convex integration [14, 12. In this degenerate form they appear for the first time in [10].

Lemma 4.1. Let $z_{0} \neq\left(0, \frac{-1}{2}\right) \in B_{1}$. Then there exists $\delta=$ $\delta\left(z_{0}\right)$ such that every $A \in \mathbb{U}$ with $\left|A-\left(0,0, z_{0}\right)\right| \leq \delta$ is the center of mass of a T4 configuration in $K$. The $T_{i}(A)$ are of the form $\left(1, x_{1}, x_{1}\right),\left(1, x_{2}, x_{2}\right),\left(-1, y_{1},-y_{1}\right),\left(-1, y_{2},-y_{2}\right) \in K$, where $\left(x_{1}, x_{2}, y_{1}, y_{2}\right)(A): \mathbb{R}^{5} \rightarrow \mathbb{R}^{2}$ are differentiable submersions. Moreover $A \in \operatorname{int}\left\{T_{i}(A)\right\}^{c}$.

Proof. First we observe that by the definition of the cone $\Lambda$

$$
(\rho, w, z)-(1, x, x) \in \Lambda \Longleftrightarrow x-w \in S_{1-\rho}
$$

and

$$
(\rho, w, z)-(-1, y,-y) \in \Lambda \Longleftrightarrow y-w \in S_{-1-\rho},
$$

if in addition we have

$$
(\rho, w, z) \in \operatorname{int}\left(\left\{\left(1, x_{1}, x_{1}\right),\left(1, x_{2}, x_{2}\right),\left(-1, y_{1},-y_{1}\right),\left(-1, y_{2},-y_{2}\right)\right\}^{c}\right)
$$

the lemma is proved. 


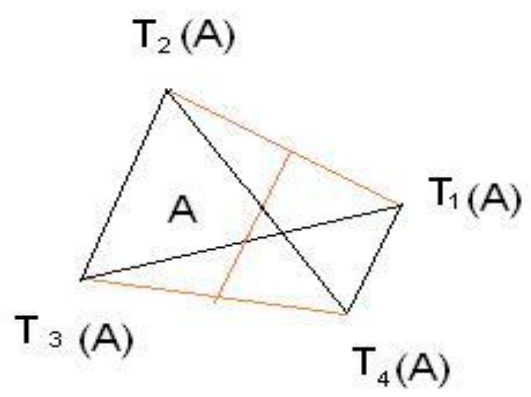

Figure 2. Convex hull of $T_{i}(A)$.

First, we notice that for each $(\rho, w, z)$ with $|\rho|<1$ there exists differentiable functions $x, y: \mathbb{R} \times \mathbb{R}^{2} \times \mathbb{R}^{2} \rightarrow \mathbb{R}^{2}$ such that

$$
(\rho, w, z) \in[(1, x, x),(-1, y,-y)] .
$$

Namely for $t=\frac{1+\rho}{2}$ and

$$
x(A)=x(\rho, w, z)=\frac{z+w}{1+\rho}, \quad y(A)=y(\rho, w, z)=\frac{w-z}{1-\rho},
$$

it holds that

$$
(\rho, w, z)=(t(1)+(1-t)(-1), t x+(1-t)(y), t x+(1-t)(-y)) .
$$

Now we consider the balls $B_{x}=w+B_{1-\rho}, B_{y}=w+B_{-1-\rho}$ with centers $a_{x}, a_{y}$.

Thus, if $\delta \leq \delta\left(z_{0}\right)$ is sufficiently small it follows that

$$
x=\frac{z+w}{1+\rho} \in B_{x} \backslash a_{x}, \quad y=\frac{z-w}{1-\rho} \in B_{y} \backslash a_{y} .
$$

In this case we can use the convexity of Euclidean balls to show that $x(A) \in\left[x_{1}, x_{2}\right], x_{1}, x_{2} \in w+S_{1-\rho}$ and similarly for $y(A)$. Namely if $|x-a| \leq r$ then

$$
x=\frac{1}{2}\left(1-\frac{|x-a|}{r}\right) \underbrace{\left(a-r \frac{x-a}{|x-a|}\right.}_{x_{1}})+\frac{1}{2}\left(1+\frac{|x-a|}{r}\right) \underbrace{\left(a+r \frac{x-a}{|x-a|}\right.}_{x_{2}}) .
$$


The same argument provides us the mapping $y_{1}(A), y_{2}(A)$ Thus we declare

$$
\begin{aligned}
& T_{1}(A)=\left(1, x_{1}, x_{1}\right), T_{2}(A)=\left(1, x_{2}, x_{2}\right), \\
& T_{3}(A)=\left(-1, y_{1},-y_{1}\right), T_{4}(A)=\left(-1, y_{2}, y_{2}\right) .
\end{aligned}
$$

We have shown the existence of $\left\{\lambda_{i}\right\}_{i=1}^{4}$ such that $0<\lambda_{i}<1$, $\sum_{i=1}^{4} \lambda_{i}=1$ and

$$
A=\sum_{i=1}^{4} \lambda_{i} T_{i}(A) .
$$

Since the $T_{i}(A)$ are linearly independent and $\lambda_{i} \neq 0$ for $i=1,2,3,4$ we arrive to the desired claim

$$
A \in \operatorname{int}\left(\cup_{i} T_{i}(A)\right)^{c} .
$$

We turn to the regularity of the mappings $T_{i}(A): \mathcal{U} \rightarrow \mathbb{R}^{2}$. It is enough to consider the case $T_{1}$. Notice that from the convexity argument follows

$$
a_{x}=a(w, \rho)=w+(1-\rho)\left(0, \frac{-1}{2}\right) \quad \text { and } \quad r=1-\rho .
$$

Thus, both are differentiable functions of $A$. Since $x \neq a_{x}$ the same is true for $x_{1}, x_{2}, y_{1}$ and $y_{2}$. It remains to see that they are submersions. It is enough to argue for the function $x_{1}$. We write $x_{1}=F_{1} \circ F_{2}$ with $F_{1}: \mathbb{R} \rightarrow \mathbb{R}^{5}, F_{1}(r, z, w)=\left(r_{x}, a_{x}, x\right)$ which are defined in $(7,6)$ and $F_{2}: \mathbb{R}^{5} \rightarrow \mathbb{R}^{2}$ is given by $F_{2}(r, a, x)=a-r \frac{x-a}{|x-a|}$. For small $\delta F_{1}$ is a submersion and $F_{2}$ is always a submersion.

Remark 4.1. It is instructive to realize that by definition if $(\rho, w, z)$ belongs to $K \cap \Lambda$ then $z_{2} \leq 0$ and thus $(0,0,0) \notin \operatorname{int}(K \cap \Lambda)^{c}$. This shows that the above strategy never could work with $z=0$ (compare with Proposition 5.1).

We will choose a set $\mathcal{U}$ adapted to the $T 4$ configuration.

Definition 4.2. Let $K \subset \mathbb{U}$. The first Lambda convex hull of $K, K^{1, \Lambda}$ is defined by

$K^{1, \Lambda}=\{A \in \mathbb{U}: A=t B+(1-t) C, \quad B, C \in K, \quad B-C \in \Lambda, t \in[0,1]\}$.

Definition 4.3. Let $z \neq\left(0, \frac{-1}{2}\right) \in B_{1}$ and the corresponding $\delta(z)$ from lemma 4.1 . We denote by $\mathbb{B}_{z} \in \mathbb{U}$ the euclidean ball centered in $(0,0, z)$ and with radius $\delta(z)$. Furthermore we declare

$$
\mathcal{U}_{z}=\cup_{i=1}^{4}\left\{\mathbb{B}_{z} \cup T_{i}\left(\mathbb{B}_{z}\right)\right\}^{1, \Lambda} \quad \text { and } \quad K_{z}=\cup_{i=1}^{4}\left\{T_{i}\left(\mathbb{B}_{z}\right)\right\} \in K .
$$

Lemma 4.2. The set $\mathcal{U}_{z} \backslash K$ is open in $\mathbb{U}$. 
Proof. Let $C \in \mathbb{U}$ with $|C| \leq \epsilon$ and $A_{t} \in \mathcal{U}_{z} \backslash K$. Our task is to find an $\epsilon$ so that $C_{t}=A_{t}+C \in \mathcal{U}_{z} \backslash K$. By definition $A_{t}=t A+(1-t) X_{A}$, where $A \in \mathbb{B}_{z}, 0<t<1$ and $X_{A} \in T_{i}\left(\mathbb{B}_{z}\right)$ for some $i=1,2,3,4$. Moreover it holds that $A-X_{A} \in \Lambda$.

It will be enough to show that if $\epsilon$ is small enough, then there exists $X \in T_{i}\left(\mathbb{B}_{z}\right)$ such that

$$
C_{t}-X \in \Lambda \quad \text { and } \quad X+\frac{1}{t}\left(C_{t}-X\right) \in \mathbb{B}_{z} .
$$

We use coordinates $A=(\rho, w, z), X_{A}=\left(1, x_{A}, x_{A}\right), A_{t}=\left(\rho_{t}, w_{t}, z_{t}\right)$, $C=\left(\rho_{C}, w_{C}, z_{C}\right)$. Since $X_{A}-A \in \Lambda$ it holds that $X_{A}-A_{t} \in \Lambda$ as well. Thus, by the definition of $\Lambda, x_{A}-w_{t} \in S_{1-\rho_{t}}$. Therefore there exists $\xi$ with $|\xi|=1$ such that

$$
X_{A}=w_{t}+\left(1-\rho_{t}\right)\left[\left(0,-\frac{1}{2}\right)+\xi\right] .
$$

Next recall that $C_{t}-(1, x, x) \in \Lambda$ if $x-w_{t}-w_{C} \in S_{1-\left(\rho_{t}+\rho_{C}\right)}$. Set $x=w_{t}+w_{C}+\left(1-\rho_{t}-\rho_{C}\right)\left(\left(0, \frac{-1}{2}\right)+\xi\right)$ and notice that

$$
\left|x-x_{A}\right| \leq\left|w_{C}\right|+2\left|\rho_{C}\right| \leq 3|C| \leq 3 \epsilon \text {. }
$$

The function $x: A \rightarrow x(A)$ is a submersion by Lemma 4.1 and thus $x\left(\mathbb{B}_{z}\right)$ is an open subset of $\mathbb{R}^{2}$. Hence, by choosing $6 \epsilon \leq$ $\operatorname{dist}\left(x_{A}, \partial x\left(\mathbb{B}_{z}\right)\right)$, we obtain that $(1, x, x) \in T_{i}\left(\mathbb{B}_{z}\right)$.

Now since $A=X_{A}+\frac{1}{t}\left(A_{t}-X_{A}\right)$ we have

$$
\begin{aligned}
\left|X+\frac{1}{t}\left(C_{t}-X\right)-A\right| & =\left|X-T_{i}(A)\right|+\frac{1}{t}\left|C_{t}-A_{t}\right|+\left|X-T_{i}(A)\right| \\
& \leq\left(3+\frac{4}{t}\right) \epsilon .
\end{aligned}
$$

Then if $\epsilon \leq \frac{1}{8} t \cdot \operatorname{dist}\left(A, \partial \mathbb{B}_{z}\right)$ it follows that $A_{C}=X+\frac{1}{t}\left(C_{t}-X\right) \in \mathbb{B}_{z}$ and

$$
C_{t}=t A_{C}+(1-t) X \in \mathcal{U}_{z}
$$

A final choice $8 \epsilon \leq \min \left\{t \cdot \operatorname{dist}\left(A, \partial \mathbb{B}_{z}\right)\right.$, $\left.\operatorname{dist}\left(x_{A}, \partial x\left(\mathbb{B}_{z}\right)\right)\right\}$ yields the claim.

\section{From GeOMEtric STRUCTURE TO WEAK SOLUTIONS}

There are many ways to pass from solutions in $\mathcal{U}$ to solutions in $K$ depending on the geometry of the sets. We have followed to present an approach which in some sense axiomatize the arguments in [6]. However it is by no means the only argument and for example one can verify that our sets $K$ and $\mathcal{U}$ verify the conditions in [10, Proposition 4.42]. The argument is easier to explain via the following definition which is related to the notion of stability near $K$ given in [10, Definition 3.15] 
Definition 5.1 (Analytic Perturbation Property, APP). The sets $K \subset$ $\mathcal{U}$ have the analytic perturbation property if for each $A \in \mathcal{U}$ and for every domain $\Omega \subset \mathbb{R}_{x}^{2} \times \mathbb{R}$ there exists a sequence $Z_{j}$ of piecewise smooth function such that

i) $\mathcal{L}\left(Z_{j}\right)=0$.

ii) $Z_{j}$ is supported in $\Omega$.

iii) $A+Z_{j} \in \mathcal{U}$ a.e.

iv) $Z_{j} \rightarrow 0$.

v) There exists $c>0$ such that $\int_{\Omega}\left|Z_{j}\right| \geq c \cdot \operatorname{dist}_{K}(A)|\Omega|$.

Next we prove that our sets enjoy the analytic perturbation property. The reader that is familiar with convex integration will realize that in practice our arguments are related to the concept of in approximation [14, 9] for $\Lambda$ convexity (based on degenerated $T 4$ configurations).

Lemma 5.1. The sets $K_{z}$ and $\mathcal{U}_{z}$ have the APP property.

Proof. We will obtain v) as a consequence of

v́) There exists $c_{1}, c_{0}>0$ such that

$$
\left|\left\{(x, t) \in \Omega:\left|Z_{j}(x, t)\right| \geq c_{1} \operatorname{dist}_{K}(A)\right\}\right| \geq c_{0}|\Omega| .
$$

Let $C \in \mathbb{B}_{z}$. By lemma 4.1 is the center of a $T 4$ configuration $\left\{T_{i}(C)\right\}_{i=1}^{4}$. Now observe that for $s$ small enough $C$ belongs also to a $T 4$ configuration supported in $T_{i, s}(C)=s C+(1-s) T_{i}(C) \in$ $\operatorname{int} K_{z, \delta}$. Namely, since $T_{i, s}(C) \rightarrow T_{i}(C)$ as $s \rightarrow 0$ it follows that $C \in \operatorname{int}\left(\left\{T_{i}(C)\right\}\right)^{c}$ implies that $C \in\left\{T_{i, s}(C)\right\}^{c}$. The other properties are obvious.

Let $T_{i, s}(C)$ be as above. First notice that since $\left|C-T_{i}(C)\right| \geq$ $\operatorname{dist}\left(C, K_{z}\right)$ by continuity we can choose $s_{0}$ such that for $s>s_{0}$ it holds that

$$
2\left|C-T_{i, s}(C)\right| \geq \operatorname{dist}\left(A, K_{z}\right) .
$$

Since $C \in\left\{T_{i, s}(C)\right\}^{c}$, there exist $\lambda_{i}>0$ with $\sum \lambda_{i}=1$ such that $C=\sum_{i} \lambda_{i} T_{i, s}(C)$. Since the $T 4$ configuration is degenerate, if we set $C_{i}^{\epsilon}=C+\epsilon \sum_{j=1}^{i}\left(T_{i, s}(C)-C\right)$ and $T_{i}^{\epsilon}(C)=T_{i, s}(C)+C_{i}^{\epsilon}$ it follows that

$$
C_{i+1}^{\epsilon}=\left(1-\frac{\epsilon}{1+\epsilon}\right) C_{i}^{\epsilon}+\frac{\epsilon}{1+\epsilon} T_{i+1}^{\epsilon}(C),
$$

with $T_{i+1}^{\epsilon}(C)-C_{i}^{\epsilon} \in \Lambda$, i.e $T_{i}^{\epsilon}$ is a non degenerate $T 4$ (We drop the dependence of $s$ for simplicity).

Fix a sequence $\eta_{j}$ such that $\Pi_{r=1}^{\infty}\left(1-\eta_{r}\right) \geq \frac{1}{2}$ and $\varepsilon=\frac{\epsilon}{1+\epsilon}>0$. We will obtain the sequence $\left\{Z_{j}\right\}_{j=0}^{\infty}$ recursively. We claim that given $Z_{j}$ there exists $Z_{j+1}$ such that

i) $\left|\left\{(x, t):\left|Z_{j+1}(x)\right| \geq \frac{1}{4} \operatorname{dist}_{K}(C) \mid\right\}\right| \geq \mid\left\{(x, t):\left|Z_{j}(x)\right| \geq\right.$ $\left.\frac{1}{4} \operatorname{dist}_{K}(C) \mid\right\} \mid+(1-\varepsilon)^{j} \varepsilon \frac{1}{2}$. 
ii) There exists $i=i(j)$ such that

$$
\Pi_{r=1}^{j}\left(1-\eta_{r}\right)(1-\varepsilon)^{j} \leq\left|\left\{(x, t): C+Z_{j+1}(x)=C_{i}^{\epsilon}\right\}\right| .
$$

iii) $Z_{j} \rightarrow 0$.

iv) $C+Z_{j} \in \mathcal{U}_{z}$.

The proof follows by induction with $Z_{0}=0$. We start by considering the open set $\Omega_{j}=\left\{(x, t) \in \Omega: C+Z_{j}=C_{i}^{\epsilon}\right\}$. Since $C_{i}^{\epsilon}=(1-$ $\left.\frac{\epsilon}{1+\epsilon}\right) C_{i-1}^{\epsilon}+\frac{\epsilon}{1+\epsilon} T_{i}^{\epsilon}(C)$ we apply the Lemma 3.3 in $\Omega_{j}$ with $\eta<\eta_{j+1}<\eta_{0}$ to obtain a new sequence $\left\{Z_{j, k}\right\}$.

- $Z_{j, k} \rightarrow 0$.

- $\left|\left\{(x, t) \in \Omega_{j}: Z_{j, k}+C_{i}^{\epsilon}=T_{i}^{\epsilon}(C)\right\}\right| \geq\left(\frac{\epsilon}{1+\epsilon}\right)\left(1-\eta_{j+1}\right)\left|\Omega_{j}\right|$.

- $\left|\left\{(x, t) \in \Omega_{j}: Z_{j, k}+C_{i}^{\epsilon}=C_{i-1}^{\epsilon}\right\}\right| \geq\left(\frac{1}{1+\epsilon}\right)\left(1-\eta_{j+1}\right)\left|\Omega_{j}\right|$.

- $\sup _{(x, t) \in \Omega_{j}} \operatorname{dist}\left(Z_{j, k}(x, t)+C_{i}^{\epsilon},\left[T_{i}^{\epsilon}(C), C_{i-1}^{\epsilon}\right]\right) \leq\left(1-\eta_{j}\right)$.

Now set $Z_{j+1, k}=Z_{j}+Z_{j, k}$ and $\varepsilon=\frac{\epsilon}{1+\epsilon}$. Let us verify properties $i)$ and $i i)$. Notice that if $(x, t) \in \Omega_{j}$ and $Z_{j, k}(x)+C_{i}^{\epsilon}=T_{i}^{\epsilon}(C)$, then $Z_{j+1, k}(x, t)=T_{i}^{\epsilon}(C)-C$ and thus by (8), for small $\epsilon$, we get

$$
\left|Z_{j+1, k}(x, t)\right| \geq \frac{1}{4} \operatorname{dist}_{K}(C) .
$$

For $i$ ) we have

$$
\begin{aligned}
\left|\left\{(x, t):\left|Z_{j+1, k}(x, t)\right| \geq \operatorname{dist}_{K}(C)\right\}\right| & \geq\left|\left\{(x, t) \in \Omega \backslash \Omega_{j}:\left|Z_{j}(x, t)\right| \geq \operatorname{dist}_{K}(C)\right\}\right| \\
& +\left|\left\{(x, t) \in \Omega_{j}: Z_{j, k}(x, t)+C_{i}^{\epsilon}=T_{i}^{\epsilon}(C)\right\}\right| \\
& \geq\left|\left\{(x, t) \in \Omega \backslash \Omega_{j}:\left|Z_{j}(x, t)\right| \geq \operatorname{dist}_{K}(C)\right\}\right| \\
& +\varepsilon\left(1-\eta_{j+1}\right)\left|\Omega_{j}\right| \\
& \geq\left|\left\{(x, t) \in \Omega \backslash \Omega_{j}:\left|Z_{j}(x, t)\right| \geq \operatorname{dist}_{K}(C)\right\}\right| \\
& +\varepsilon \prod_{r=1}^{j+1}\left(1-\eta_{r}\right)(1-\varepsilon)^{j}|\Omega| \\
\geq \frac{1}{2} \varepsilon(1-\varepsilon)^{j}|\Omega| . &
\end{aligned}
$$

As for $i i)$, we have

$$
\begin{aligned}
& \left|\left\{(x, t) \in \Omega: Z_{j+1, k}(x, t)+C=C_{i-1}^{\epsilon}\right\}\right| \\
& \geq \mid\left\{(x, t) \in \Omega_{j}: Z_{j}(x, t)+Z_{j, k}(x, t)+C=C_{i-1}^{\epsilon}\left|\geq(1-\varepsilon)\left(1-\eta_{j+1}\right)\right| \Omega_{j} \mid\right. \\
& \geq(1-\varepsilon)^{j+1} \Pi_{r=1}^{j+1}\left(1-\eta_{j+1}\right)|\Omega| .
\end{aligned}
$$

Next, property iv) follows because the segments $\left[T_{i}^{\epsilon}(C), C_{i-1}^{\epsilon}\right]$ are compact subsets of $\mathcal{U}_{z}$ and by a diagonal argument we choose a subsequence $Z_{j, k(j)}$ such that

$$
Z_{j} \rightarrow 0 .
$$


It just remains to show that $Z_{j}$ satisfies property $\mathrm{v}$ in the definition of analytic perturbation property (APP). Indeed by property i) in the definition of $Z_{j}$ yields

$$
\begin{aligned}
\left|\left\{(x, t) \in \Omega:\left|Z_{j+1}(x, t)\right| \geq \frac{1}{4} \operatorname{dist}_{K}(C)\right\}\right| & =|\Omega| \sum_{k=1}^{j}(1-\epsilon)^{j} \epsilon \frac{1}{2}=|\Omega| \frac{1}{2}\left(1-\epsilon^{j+1}\right) \\
& \geq \frac{1}{4}|\Omega|
\end{aligned}
$$

for $j$ large enough. Thus for $C \in \mathbb{B}_{z}$ (APP) holds.

Let $A \in \mathcal{U}_{z} \backslash \mathbb{B}_{z}$. By definition $A=s C+(1-s) X_{C}$ with $C \in \mathbb{B}_{z}$, $X_{C} \in T_{i}\left(\mathbb{B}_{z}\right), 0<s<1$ and $C-X_{C} \in \Lambda$. Consider the sequence $\left\{Z_{j}\right\}$ given by Lemma 3.3. If $s<\frac{7}{8}\left\{Z_{j}\right\}$ is the sequence required by the (APP) property. Hence we may assume that $s \geq \frac{7}{8}$, obtaining that $\left|\left\{x \in \Omega: A+Z_{j}(x)=C\right\}\right| \geq \frac{7}{8}$. Let $\Omega_{j}=\left\{(x, t) \in \Omega: A+Z_{j}(x, t)=\right.$ $C\}$. Since $C \in \mathbb{B}_{z}$ we can use the argument above for the domain $\Omega_{j}$. We are provided with a sequence $\left\{Z_{k}\right\}$ such that $Z_{k} \rightarrow 0, Z_{k}+C \in \mathcal{U}_{z}$ and

$$
\left|\left\{(x, t) \in \Omega_{j}:\left|Z_{k}(x, t)\right| \geq \frac{1}{4} \operatorname{dist}_{K}(C)\right\}\right| \geq \frac{1}{4}\left|\Omega_{j}\right| \geq \frac{7}{32}|\Omega| .
$$

A proper subsequence $W_{j}=Z_{j}+Z_{k(j)}$ satisfies all the desired properties in the definition of (APP). As a matter of fact just property $\dot{v}$ needs verification. Notice that for $C \in \mathbb{B}_{z}$ and $X \in T_{i}\left(\mathbb{B}_{z}\right)$ it holds that

$$
1-\delta<|C-X| \leq 1+\delta+\max _{C \in \mathbb{B}_{z}}\left|T_{i}(C)\right|=M(\delta) .
$$

Thus $\operatorname{dist}_{K}(C)>1-\delta$. Next we notice that

$$
\operatorname{dist}_{K}(A) \leq\left|A-X_{C}\right| \leq s\left|C-X_{C}\right| \leq M(\delta) .
$$

Let $(x, t) \in \Omega_{j}$ be such that $\left|Z_{k}(x, t)\right| \geq \frac{1}{4} \operatorname{dist}_{K}(C)$. Then

$$
\begin{aligned}
\left|W_{j}(x, t)\right| & \geq \frac{1}{4} \operatorname{dist}_{K}(C)-|C-A| \\
& \geq \frac{1}{4}(1-\delta)-(1-s)\left|C-X_{C}\right| \\
& \geq \frac{1}{8}(1-\delta) .
\end{aligned}
$$

Hence if we declare $c_{1}(\delta)=\frac{1-\delta}{8 M(\delta)}$, and we put together $(9)$ and 10 we obtain

$$
c_{1}(\delta) \operatorname{dist}_{K}(A) \leq\left|W_{j}(x, t)\right|
$$

for every $(x, t) \in \Omega_{j}$ with $\left|Z_{k}(x, t)\right| \geq \frac{1}{4} \operatorname{dist}_{K}(C)$. We arrive to the estimate 


$$
\left|\left\{(x, t) \in \Omega:\left|W_{j}(x, t)\right| \geq c_{1}(\delta) \operatorname{dist}_{K}(A) \mid\right\} \geq \frac{7}{32}\right| \Omega \mid .
$$

Finally ( $\left.\mathrm{v}^{\prime}\right)$ holds with $c_{1}(\delta)=c_{1}$ and $c_{0}=\frac{7}{32}$.

Next we show that if a set has a perturbation property there are many solutions for our inclusion. We formulate the existence theorem in the following way:

Theorem 5.1. Let $K, \mathcal{U}$ be two sets that satisfy the Analytic Perturbation Property and $\Omega \subset \mathbb{T}^{2} \times \mathbb{R}$ a bounded open set and $A \in \mathcal{U}$. Then there are infinitely many solutions $U$ such that

(1) $\mathcal{L}(U)=0$

(2) $U(x, t) \in K$ a.e $(x, t) \in \Omega$

(3) $U(x, t)=A$ for $(x, t) \in \mathbb{T}^{2} \times \mathbb{R} \backslash \Omega$

We shall prove the theorem using Baire category since it yields infinitely many solutions. In addition we will provide a direct argument to construct such solutions since it helps to understand its nature.

Definition 5.2 (The space of subsolutions $X_{0}$ ). Let $A \in \mathcal{U}$. We say that $U \in L^{\infty}\left(\mathbb{T}^{2} \times \mathbb{R}\right)$ belongs to $X_{0}$ if

(I) $U$ is piecewise smooth.

(Regularity)

(II) For $t \leq 0$ and $t \geq T U(x, t)=A$.

(III) $\mathcal{L}(U)=0$.

(Boundary conditions)

(IV) $U(x, t) \in \mathcal{U}$ a.e $(x, t) \in \mathbb{T}^{2} \times \mathbb{R}$.

(Conservation law)

(Relaxed inclusion)

We endow $X_{0}$ with the $L^{\infty}$ weak star topology and declare $X=\overline{X_{0}}$. If a set has the APP property then it holds the perturbation lemma.

Lemma 5.2 (Perturbation Lemma). There exists $C>0$ such that for every $U_{0} \in X_{0}$ we can find a sequence $U_{k} \in X_{0}$ such that $U_{k} \rightarrow U$ but

$$
\int_{\mathbb{T}^{2} \times \mathbb{R}}\left|U_{k}-U_{0}\right|^{2} \geq C \int_{\mathbb{T}^{2} \times[0, T]} \operatorname{dist}_{K}^{2}\left(U_{0}\right)
$$

Proof. The sequence $U_{k}$ will differ from $U_{0}$ only on the compact set $\mathbb{T}^{2} \times[0, T]$. Since $U_{0}$ is piecewise smooth we can find a finite family of pairwise disjoint balls $B_{j} \Subset \mathbb{T}^{2} \times[0, T]$ such that

$$
\int_{\mathbb{T}^{2} \times[0, T]} \operatorname{dist}_{K}\left(U_{0}\right) \leq 2 \sum \operatorname{dist}_{K}\left(U_{0}\left(x_{j}, t_{j}\right)\left|B_{j}\right|\right.
$$

Next we use that $U_{0}\left(x_{j}, t_{j}\right) \in \mathcal{U}_{z}$ to apply the APP property in the domain $B_{j}$. Let us called the corresponding perturbation sequence by $\left\{Z_{j, k}\right\}_{k=1}^{\infty}$ supported in $B_{j}$. Thus we consider the sequence

$$
U_{k}=U_{0}+\sum Z_{j, k}
$$

which satisfies that $U_{k} \in \mathcal{U}$ (a small continuity argument is needed). 
Then

$$
\begin{aligned}
\int_{\mathbb{T}^{2} \times \mathbb{R}}\left|U_{k}-U_{0}\right|= & \sum_{j} \int_{B_{j}}\left|Z_{j, k}\right| \geq c \sum \operatorname{dist}_{K}\left(U_{0}\left(x_{j}, t_{j}\right)\right)\left|B_{j}\right| \\
& \geq \frac{c}{2} \int \operatorname{dist}_{K}\left(U_{0}\right)
\end{aligned}
$$

where the last inequality follows from (11).

Next we recall how to obtain a solution $U(x, t) \in K$ a.e. from the perturbation lemma and the non emptiness of $X_{0}$.

The solution $U$ will be the strong limit of a sequence $U_{k}$ which is obtained from the perturbation lemma. Hence strong convergence implies that $U$ is in $K$. The Baire category argument yields the existence of such $U$ as point of continuity of the Identity map and then a sequence $U_{k}$ which converge to it. The direct argument builds the sequence $U_{k}$ iteratively and then show that indeed it converges strongly to some $U$.

\section{Baire Category:}

We consider the Identity as a Baire-one map from $(X$, weak $*)$ to $\left(X, L^{1}\left(\mathbb{T}^{2} \times \mathbb{R}\right)\right)($ see [6, Lemma 4.5]).

Lemma 5.3. Let $U \in X$ be a point of continuity of the identity. Then $U \in K$ a.e $(x, t) \in \Omega \times[0, T]$.

Suppose that $U \in X$ is a point of continuity of the identity. By definition of $X$, there exists $\left\{U_{j}\right\} \in X_{0}$ such that

$$
U_{j} \rightarrow U
$$

in the weak star topology. As $u$ is a point of continuity of the identity it holds that

$$
\lim _{j \rightarrow \infty} \int_{\mathbb{T}^{2} \times \mathbb{R}}\left|U_{j}-U\right|=0
$$

and thus,

$$
\lim _{j \rightarrow \infty} \int_{\mathbb{T}^{2} \times[0, T]} \operatorname{dist}_{K}\left(U_{j}\right) d x=\int_{\mathbb{T}^{2} \times[0, T]} \operatorname{dist}_{K}(U) .
$$

For each $U_{j}$ we use a perturbation lemma. The corresponding sequence is indexed by $U_{j, k}$. By a diagonal argument we can choose a sequence $U_{j, k(j)}$ such that

$$
U_{j, k(j)} \rightarrow U
$$

and since $U$ is a point of continuity yields

$$
\lim _{j \rightarrow \infty} \int_{\mathbb{T}^{2} \times \mathbb{R}}\left|U_{j, j(k)}-U\right|=0
$$

Now 


$$
\begin{aligned}
\int_{\mathbb{T}^{2} \times[0, T]} \operatorname{dist}_{K}(U) & =\lim _{j \rightarrow \infty} \int_{\mathbb{T}^{2} \times[0, T]} \operatorname{dist}_{K}\left(U_{j}\right) \underbrace{\leq}_{P . \text { Lemma }} C \lim _{j \rightarrow \infty} \int_{\mathbb{T}^{2} \times \mathbb{R}_{t}}\left|U_{j}-U_{j, k(j)}\right| \\
& \leq C \lim _{j \rightarrow \infty} \int_{\mathbb{T}^{2} \times \mathbb{R}}\left(\left|U_{j}-U\right|+\left|U-U_{j, k(j)}\right|\right)=0 .
\end{aligned}
$$

Proof of Theorem 5.1: It is a well known fact that the points of continuity of a Baire one map are a set of second category (see [17]. Therefore there are infinitely many $U \in X$ such that $U \in K$ a.e. and $(x, t) \in \Omega$. This is equivalent to property (II) in Theorem 5.1. Since (I) and (III) hold for any element in $X_{0}$ they hold as well for any $U \in X$.

\section{Direct Construction:}

Lemma 5.4. To each function $U_{0} \in X_{0}$ we can associate a sequence $\left\{U_{j}\right\}_{j=0}^{\infty} \in X_{0}$ such that there exists $U_{\infty} \in X$ satisfying

- $U_{\infty} \in K$ a.e. $(x, t) \in \mathbb{T}^{2} \times[0, T]$

- $\lim _{j \rightarrow \infty} \int_{\mathbb{T}^{2} \times \mathbb{R}}\left|U_{j}-U_{\infty}\right|=0$

Proof. We construct directly a function $U_{\infty}$ in $X$ and a sequence $U_{k} \in$ $X$ which converges strongly to $U$ as follows. We start with $U_{0}$ in the lemma and obtain with $U_{k+1}$ from $U_{k}$ by the perturbation lemma. To each $U_{k}$ we associate a mollifier $\eta_{j(k)}$ such that

$$
\int_{\mathbb{T}^{2} \times \mathbb{R}}\left|U_{k}-\eta_{j(k)} * U_{k}\right| \leq 2^{-k}
$$

Then we apply the perturbation lemma to the function $U$ to obtain a corresponding sequence $U_{s} \in X$. By the weak convergence property we can choose $s(k)$ so that for each $i<k$

$$
\left.\int_{\mathbb{T}^{2} \times \mathbb{R}} \mid U_{k}-U_{s(k)} * \eta_{j(i)}\right) \mid \leq 2^{-k}
$$

and finally we declare $U_{k+1}=U_{s(k)}$. Properties $(12)$ and $(13)$ yield strong convergence. Now

$$
\begin{aligned}
& \int_{\mathbb{T}^{2} \times \mathbb{R}} \operatorname{dist}_{K}(U)=\lim _{k \rightarrow \infty} \int_{\mathbb{T}^{2} \times \mathbb{R}} \operatorname{dist}_{K}\left(U_{k}\right) \\
& \underbrace{\leq}_{\text {P.Lemma }} C \lim _{k \rightarrow \infty} \int_{\mathbb{T}^{2} \times \mathbb{R}}\left|U_{k}-U_{k+1}\right|=0
\end{aligned}
$$

Theorem 5.2. For every $T>0$ there exists infinitely many non trivial weak solutions $(\rho, v) \in L^{\infty}\left(\mathbb{T}^{2} \times[0, T]\right)$ to the $2 D$ IPM system (4) such that $\rho(x, 0)=0$. 
Proof. We fix $z \neq\left(0,-\frac{1}{2}\right) \in B_{1}$ and the corresponding $\mathbb{B}_{z}, \mathcal{U}_{z}$ and $K_{z}$ given by definition 4.3. By Lemma 5.1 $\mathcal{U}_{z}$ and $K_{z}$ enjoy the APP property. Thus we can apply Theorem 5.1 to these sets, the domain $\Omega$ equals $\mathbb{T}^{2} \times(0, T)$ and $A$ equals $(0,0, z)$.

We claim that each of the functions $U=(\rho, v, q) \in X$ with $U(x, t) \in$ $K_{z}$ a.e $(x, t) \in \mathbb{T}^{2} \times[0, T]$ from Theorem 5.1. yields a weak solution $(\rho, v)$ to the IPM system with initial values $\rho(x, 0)=0$. Indeed from the fact that $\mathcal{L}(\rho, v, q)=0$ it follows that $\operatorname{div}(v)=0$ and that $\operatorname{Curl}(v+$ $(0, \rho))=0$. Thus there exists a function $p$ such that $\nabla p=v+(0, \rho)$. The only delicate issue corresponds to the equation of conservation of mass where we see the nonlinearity. By definition we have that for $\psi \in C_{0}^{\infty}\left[\mathbb{T}^{2} \times \mathbb{R}\right]$

$$
0=\int_{\mathbb{T}^{2} \times \mathbb{R}}\left(\partial_{t} \psi \rho+\nabla \psi \cdot q\right) .
$$

Notice that $\varphi \in C_{c}^{\infty}\left[\mathbb{T}^{2} \times[0, T)\right]$ can be extended to $\tilde{\psi} \in C_{0}^{\infty}\left[\mathbb{T}^{2} \times \mathbb{R}\right]$. Next we observe that $q(x, t)=z$ for a.e. $(x, t) \in \mathbb{T}^{2} \times \mathbb{R} \backslash[0, T)$. Thus for a.e. $t \in \mathbb{R} \backslash[0, T)$ it holds that

$$
\int_{\mathbb{T}^{2}} \nabla \tilde{\psi}(x, t) \cdot q(x, t) d x=\int_{\mathbb{T}^{2}} \nabla \tilde{\psi}(x, t) \cdot z d x=0 .
$$

Now we plug $\tilde{\psi}$ in (5). Then (14) together with the fact $\rho(x, t)=0$ for a.e. $(x, t) \in \mathbb{T}^{2} \times \mathbb{R} \backslash[0, T)$ yields

$$
0=\int_{0}^{T} \int_{\mathbb{T}^{2}}\left(\partial_{t} \varphi \rho+\nabla \varphi \cdot q\right)=\int_{0}^{T} \int_{\mathbb{T}^{2}}\left(\partial_{t} \varphi \rho+\nabla \varphi \cdot \rho v\right),
$$

where in the last equality we have used that for $(x, t) \in \mathbb{T}^{2} \times[0, T], q=$ $\rho v$. Hence $(\rho, v)$ is a weak solution to the IPM system according to (1), (2), (3).

Remark 5.1. The boundary values (or initial data) are attained in the weak sense. Namely we use the fact (see [7]) that a solution to $\mathcal{L}(U)=0$ can be redefined in a set of times of measure zero in a way that the map

$$
t \rightarrow \int_{\mathbb{T}^{2}} v(x, t) \varphi
$$

is continuous. Since for negative $t \int_{\mathbb{T}^{2}} v(x, t) \varphi=0$ we obtain the desired

$$
\lim _{t \rightarrow 0} \int_{\mathbb{T}^{2}} v(x, t) \varphi=0
$$

and we can argue similarly for the density. From this point of view it is not surprising that $\lim _{t \rightarrow 0} \rho(x, t)=0=\lim _{t \rightarrow 0} v(x, t)$ but $\lim _{t \rightarrow 0} \rho(x, t) v(x, t)=z \neq 0$ since they are weak limits and weak limits do not commute with products. In fact from this we deduce that our solutions do not attain the boundary values in the strong sense and 
hence by the Frechet Kolmogorov theorem they are highly irregular. Another additional feature of the weak solution is that $|\rho(x, t)|=1$ for a.e. $(x, t) \in \mathbb{T}^{2} \times(0, T)$ which prevents the pointwise convergence to the initial data.

We conclude with a proposition pointing out to the fact that this method does not seem to yield a solution compact in space and time. For that we need some more standard terminology. We say that a function $f: \mathbb{U} \rightarrow \mathbb{R}$ is $\Lambda$ convex if for $A, B, C \in \mathbb{U}$ such that $A-B \in \Lambda$ the function $g(t): f(C+t(A-B))$ is convex. Let $K \subset \mathbb{U}$. Then the Lambda convex hull of $K, K^{\lambda}$

$$
K^{\Lambda}=\{A \in \mathbb{U}: f(A) \leq \sup f(C), \quad C \in K, f \quad \Lambda \text { convex }\} .
$$

In all the methods to solve differential inclusions the canonical choice for the set $\mathcal{U}$ is the interior of $K^{\Lambda}$. However in our case the set $K$ can not be there.

Proposition 5.1. The set $K \subset \partial K^{\Lambda}$

Proof. The proof relies on the fact that the function $f(A)=z_{2}-\rho v_{2}$ is $\Lambda$ convex. In fact it is easy to see that $\operatorname{det}_{\Lambda}$ is $\Lambda$ linear, thus $-\operatorname{det}_{\Lambda}$ is $\Lambda$ convex. Hence

$$
|v|^{2}-\operatorname{det}_{\Lambda}=-\rho v_{2}
$$

is $\Lambda$ convex and $f$ is $\Lambda$ convex. Moreover $f(A)=0$ for all $A \in K$ and hence $K^{\Lambda} \subset f^{-1}(-\infty, 0]$ and $K \subset \partial f^{-1}(-\infty, 0] \cap K^{\Lambda} \subset \partial K^{\Lambda}$.

Remark 5.2. The above argument also shows that if $0 \in K^{\Lambda}$ (or any other element in $K$ ) the elements of the splitting sequence in the laminate [10] should belong to $f^{-1}(0)$.

Acknowledgments. DC and FG were partially supported by MTM2008-03754 project of the MCINN (Spain) and StG203138 CDSIF grant of the ERC. DF was partially supported by MTM2008-02568 project of the MCINN (Spain). FG was partially supported by NSF-DMS grant 0901810. The authors would like to thank L. Székelyhidi Jr for helpful conversations.

\section{REFERENCES}

[1] Bear, J. Dynamics of Fluids in Porous Media. American Elsevier, Boston, MA, 1972.

[2] Constantin, P., E, W., And Titi, E. S. Onsager's conjecture on the energy conservation for solutions of Euler's equation. Comm. Math. Phys. 165, 1 (1994), 207-209.

[3] Córdoba, D., And Gancedo, F. Contour dynamics of incompressible 3-D fluids in a porous medium with different densities. Comm. Math. Phys. 273, 2 (2007), 445-471. 
[4] Córdoba, D., Gancedo, F., and Orive, R. Analytical behavior of twodimensional incompressible flow in porous media. J. Math. Phys. 48, 6 (2007), 065206, 19.

[5] Dacorogna, B., And Marcellini, P. General existence theorems for Hamilton-Jacobi equations in the scalar and vectorial cases. Acta Math. 178 (1997), 1-37.

[6] De Lellis, C., And SzÉKelyhidi, JR., L. The Euler equation as a differential inclussion. Ann. Math 170, 3 (2009), 1417-1436.

[7] De Lellis, C., AND SzÉKelYhidi, JR., L. On admissibility criteria for weak solutions of the Euler equations. Arch. Rational Mech. Anal. 195, 1 (2010), $225-260$.

[8] Gancedo, F. Existence for the $\alpha$-patch model and the QG sharp front in Sobolev spaces. Adv. Math. 217, 6 (2008), 2569-2598.

[9] Gromov, M. Partial differential relations, vol. 9 of Ergebnisse der Mathematik und ihrer Grenzgebiete (3). Springer-Verlag, Berlin, 1986.

[10] Kirchneim, B. Rigidity and Geometry of microstructures. Habilitation thesis, University of Leipzig, 2003.

[11] Kirchheim, B., Müller, S., And Šverák, V. Studying nonlinear PDE by geometry in matrix space. In Geometric analysis and Nonlinear partial differential equations, S. Hildebrandt and H. Karcher, Eds. Springer-Verlag, 2003, pp. 347-395.

[12] Kirchheim, B., And Preiss, D. Construction of Lipschitz mappings having finitely many gradients without rank-one connections. in preparation.

[13] MajdA, A. J., AND BerTOzZI, A. L. Vorticity and incompressible flow, vol. 27 of Cambridge Texts in Applied Mathematics. Cambridge University Press, Cambridge, 2002.

[14] Müller, S., AND ŠverÁK, V. Convex integration for Lipschitz mappings and counterexamples to regularity. Ann. of Math. (2) 157, 3 (2003), 715-742.

[15] Müller, S., And Sychev, M. A. Optimal existence theorems for nonhomogeneous differential inclusions. J. Funct. Anal. 181 (2001), 447-475.

[16] Onsager, L. Statistical hydrodynamics. Nuovo Cimento (9) 6, Supplemento, 2(Convegno Internazionale di Meccanica Statistica) (1949), 279-287.

[17] Охтову, J. C. Measure and category, second ed., vol. 2 of Graduate Texts in Mathematics. Springer-Verlag, New York, 1980.

[18] Pedregal, P. Laminates and microstructure. European J. Appl. Math. 4 (1993), 121-149.

[19] Pedregal, P. Parametrized measures and variational principles, vol. 30 of Progress in Nonlinear Differential Equations and their Applications. Birkhäuser Verlag, Basel, 1997.

[20] Resnick, S. Dynamical problems in nonlinear advective partial differential equations. Dissertation, University of Chicago, 1995.

[21] Rodrigo, J. L. On the evolution of sharp fronts for the quasi-geostrophic equation. Comm. Pure Appl. Math. 58, 6 (2005), 821-866.

[22] Scheffer, V. An inviscid flow with compact support in space-time. J. Geom. Anal. 3, 4 (1993), 343-401.

[23] Shnirelman, A. On the nonuniqueness of weak solution of the Euler equation. Comm. Pure Appl. Math. 50, 12 (1997), 1261-1286.

[24] SzÉKELYHIDI, JR., L. The regularity of critical points of polyconvex functionals. Arch. Ration. Mech. Anal. 172, 1 (2004), 133-152.

[25] TARTAR, L. Compensated compactness and applications to partial differential equations. In Nonlinear analysis and mechanics: Heriot-Watt Symposium, Vol. IV, vol. 39 of Res. Notes in Math. Pitman, Boston, Mass., 1979, pp. 136-212. 
[26] TARTAR, L. The compensated compactness method applied to systems of conservation laws. In Systems of nonlinear partial differential equations (Oxford, 1982), vol. 111 of NATO Adv. Sci. Inst. Ser. C Math. Phys. Sci. Reidel, Dordrecht, 1983, pp. 263-285.

[27] Wu, J. The quasi-geostrophic equation and its two regularizations. Comm. Partial Differential Equations 27, 5-6 (2002), 1161-1181.

Diego Córdoba, Instituto de Ciencias Matemáticas, Consejo SupeRior de Investigaciones Cientificas, Calle Serrano 123, 28006 Madrid, SPAIN.

E-mail address: dcg@icmat.es

Daniel Faraco, Instituto de Ciencias Matemáticas CSIC-UAM-UCMUC3M and Department of Mathematics, Universidad Autónoma de MADRID, 28049 MADrID, Spain.

E-mail address: daniel.faraco@uam.es

Francisco Gancedo, Department of Mathematics, University of Chicago, 5734 University Avenue, Chicago, IL 60637, U.S.A.

E-mail address: fgancedo@math.uchicago.edu 